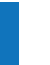

\title{
CLASSIFICATION OF $h$-HOMOGENEOUS PRODUCTION FUNCTIONS WITH CONSTANT ELASTICITY OF SUBSTITUTION
}

\author{
BANG-YEN CHEN
}

\begin{abstract}
Almost all economic theories presuppose a production function, either on the firm level or the aggregate level. In this sense the production function is one of the key concepts of mainstream neoclassical theories. There is a very important class of production functions that are often analyzed in both microeconomics and macroeconomics; namely, $h$-homogeneous production functions. This class of production functions includes two important production functions; namely, the generalized Cobb-Douglas production functions and ACMS production functions. It was proved in 2010 by L. Losonczi [12] that twice differentiable two-inputs $h$-homogeneous production functions with constant elasticity of substitution (CES) property are Cobb-Douglas' and ACMS production functions. Lozonczi also pointed out in [12] that his proof does not work for production functions of $n$-inputs with $n>2$.

In this paper we settle this classification problem completely by classifying all $h$-homogeneous production functions which satisfy the CES property. More precisely, we prove that, for arbitrary number of inputs, the only twice differentiable $h$-homogeneous production functions satisfying the CES property are the generalized Cobb-Douglas production functions and the generalized ACMS production functions.
\end{abstract}

\section{Introduction}

In microeconomics and macroeconomics, a production function is a positive nonconstant function that specifies the output of a firm, an industry, or an entire economy for all combinations of inputs. Almost all economic theories presuppose a production function, either on the firm level or the aggregate level. In this sense, the production function is one of the key concepts of mainstream neoclassical theories. By assuming that the maximum output technologically possible from a given set of inputs is achieved, economists using a production function in analysis are abstracting from the engineering and managerial problems inherently associated with a particular production process.

Let $\mathbb{R}$ denote the set of real numbers. Let us put

$$
\mathbb{R}_{+}=\{r \in \mathbb{R}: r>0\} \text { and } \mathbb{R}_{+}^{n}=\left\{\left(x_{1}, \ldots, x_{n}\right) \in \mathbb{R}^{n}: x_{1}, \ldots, x_{n}>0\right\} .
$$

2010 Mathematics Subject Classification. Primary 90A11; Secondary 91B64.

Key words and phrases. Homogeneous production function, constant elasticity of substitution, CobbDouglas production function, ACMS production function. 
In this paper, by a production function we mean a function $f: D \subset \mathbb{R}_{+}^{n} \rightarrow \mathbb{R}_{+}$from a domain $D$ of $\mathbb{R}_{+}^{n}$ into $\mathbb{R}^{+}$which has non-vanishing first derivatives.

Let $h$ be a positive number. A production function $Q=f\left(x_{1}, \cdots, x_{n}\right)$ is said to be $h$ homogeneous or homogeneous of degree $h$, if

$$
f\left(t x_{1}, \ldots, t x_{n}\right)=t^{h} f\left(x_{1}, \ldots, x_{n}\right)
$$

holds for each $t \in \mathbb{R}$ for which (1.1) is defined. A homogeneous function of degree one is called linearly homogeneous.

If $h>1$, the function exhibits increasing return to scale, and it exhibits decreasing return to scale if $h<1$. If it is homogeneous of degree 1 , it exhibits constant return to scale. The presence of increasing returns means that a one percent increase in the usage levels of all inputs would result in a greater than one percent increase in output; the presence of decreasing returns means that it would result in a less than one percent increase in output. Constant returns to scale is the in-between case.

In 1928, C. W. Cobb and P. H. Douglas [7] introduced a famous two-factor production function, nowadays called Cobb-Douglas production function, in order to describe the distribution of the national income by help of production functions.

Cobb-Douglas two-factor production function is given by

$$
Y=b L^{k} C^{1-k}
$$

where $L$ represents the labor input, $C$ is the capital input, $b$ is the total factor productivity and $Y$ is the total production. Later work in the 1940s prompted them to allow for the exponents on $C$ and $L$ vary, which resulting in estimates that subsequently proved to be very close to improved measure of productivity developed at that time $[8,10]$.

In its generalized form the Cobb-Douglas production function may be written as

$$
Q(\mathbf{x})=b x_{1}^{\alpha_{1}} \cdots x_{n}^{\alpha_{n}}
$$

for $\mathbf{x}=\left(x_{1}, \ldots, x_{n}\right) \in \mathbb{R}_{+}^{n}$, where $b$ is a positive constant and $\alpha_{1}, \ldots, \alpha_{n}$ are nonzero constants. This function $Q$ is known as the generalized Cobb-Douglas production function.

The most common quantitative indices of production factor substitutability are forms of the elasticity of substitution. The elasticity of substitution was originally introduced by J. R. Hicks [11] in case of two inputs for the purpose of analyzing changes in the income shares of labor and capital. 
R. G. Allen and J. R. Hicks [2] suggested a generalization of Hicks original two variable elasticity concept given as follows: Let $f$ be a twice differentiable production function. Put

$$
H_{i j}(\mathbf{x})=\frac{\frac{1}{x_{i} f_{x_{i}}}+\frac{1}{x_{j} f_{x_{j}}}}{-\frac{f_{x_{i} x_{i}}}{f_{x_{i}}^{2}}+\frac{2 f_{x_{i} x_{j}}}{f_{x_{i}} f_{x_{j}}}-\frac{f_{x_{j} x_{j}}}{f_{x_{j}}^{2}}}
$$

for $\mathbf{x} \in \mathbb{R}_{+}^{n}, 1 \leq i \neq j \leq n$, where the subscripts of $f$ denote partial derivatives i.e.

$$
f_{x_{i}}=\frac{\partial f}{\partial x_{i}}, f_{x_{i} x_{j}}=\frac{\partial^{2} f}{\partial x_{i} \partial x_{j}},
$$

all partial derivatives are taken at the point $\mathbf{x}$ and the denominator is assumed to be different from zero.

The $H_{i j}$ is called the Hicks elasticity of substitution of the $i$-th production variable (factor) with respect to the $j$-th production variable (factor).

A twice differentiable production function $f$ is said to satisfy the CES (constant elasticity of substitution) property if there is a nonzero constant $\sigma \in \mathbb{R}$ such that

$$
H_{i j}(\mathbf{x})=\sigma \text { for } \mathbf{x} \in \mathbb{R}_{+}^{n} \text { and } 1 \leq i \neq j \leq n .
$$

K. J. Arrow, H. B. Chenery, B. S. Minhas and R. M. Solow [3] introduced a two-factor CES production function given by

$$
Q=F \cdot\left(a K^{r}+(1-a) L^{r}\right)^{\frac{1}{r}}
$$

where $Q$ is the output, $F$ the factor productivity, $a$ the share parameter, $K, L$ the primary production factors (capital and labor), $r=(s-1) / s$, and $s=1 /(1-r)$ is the elasticity of substitution.

The generalized ACMS production function (or the generalized CES production function) is defined as

$$
Q(\mathbf{x})=b\left(\sum_{i=1}^{n} a_{i}^{\rho} x_{i}^{\rho}\right)^{\frac{h}{\rho}}, \mathbf{x}=\left(x_{1}, \ldots, x_{n}\right) \in \mathbb{R}_{+}^{n},
$$

with $a_{1}, \ldots, a_{n}, b, h>0, \rho<1, \rho \neq 0$.

It is well-known that both the generalized Cobb-Douglas production function and the ACMS production function satisfy the CES property. In fact, it is easy to verify that $H_{i j}(\mathbf{x})=1$ for the generalized Cobb-Douglas production function and $H_{i j}(\mathbf{x})=1 / \rho$ for the ACMS production function if $\rho \neq 1$. For $\rho=1$ the denominator of $H_{i j}$ is zero, hence it is not defined.

From mathematical point of view, it is a quite interesting problem to classify $h$-homogeneous production functions which satisfy the CES property. For functions of two variables, this was done recently by to L. Losonczi in 2010. More precisely, Losonczi proved the following result in [12, Theorem 10]. 
Theorem A. Suppose that $P: \mathbb{R}_{+}^{2} \rightarrow \mathbb{R}_{+}$is a twice differentiable two-variable production function, homogeneous of degree $h \neq 0$ and satisfying $H_{12}(\mathbf{x})=\sigma$ for some $0 \neq \sigma \in \mathbb{R}$. Then

$$
P(x, y)= \begin{cases}C x^{\alpha} y^{h-\alpha}, & \text { if } \sigma=1, \\ \left(\beta_{1} x^{\frac{h}{\beta}}+\beta_{2} y^{\frac{h}{\beta}}\right)^{\beta}, & \text { if } \sigma \neq 1,\end{cases}
$$

where $\alpha \neq 0$ is arbitrary nonzero constant such that $h-\alpha \neq 0$ holds, $C, \beta_{1}, \beta_{2}$ are are arbitrary positive constants.

Theorem A generalizes and somewhat clarifies analogous result of [3]. In the closing remark of [12], L. Losonczi pointed out that the method of his proof of Theorem A does not work for production functions with more than two inputs.

In this paper, we settle this problem completely, for arbitrary number of inputs, by classifying all $h$-homogeneous production functions which satisfy the CES property. More precisely, we prove the following.

Theorem 1. Let $f\left(x_{1}, \ldots, x_{n}\right)$ be a twice differentiable $h$-homogeneous production function. If $f$ satisfies the constant elasticity of substitution property, then it is either the generalized CobbDouglas production function given by

$$
f\left(x_{1}, \ldots, x_{n}\right)=b x_{1}^{\alpha_{1}} \cdots x_{n}^{\alpha_{n}}
$$

with $\alpha_{1}, \ldots, \alpha_{n} \neq 0$ and $\sum_{i=1}^{n} \alpha_{i}=h$ or the generalized ACMS production function given by

$$
f\left(x_{1}, \ldots, x_{n}\right)=b\left(\sum_{i=1}^{n} a_{i}^{\rho} x_{i}^{\rho}\right)^{\frac{h}{\rho}},
$$

with $a_{1}, \ldots, a_{n}, b, h>0, \rho<1, \rho \neq 0$.

\section{Proof of Theorem 1.}

Let $Q=f\left(x_{1}, \ldots, x_{n}\right)$ be a twice differentiable $h$-homogeneous production function. Then $f$ has non-vanishing first partial derivatives. The Euler Homogeneous Function Theorem implies that

$$
x_{1} f_{x_{1}}+x_{2} f_{x_{2}}+\cdots+x_{n} f_{x_{n}}=h f \text {. }
$$

If $h=0$, then after taking the partial derivatives of (1.1) with respect to $x_{1}, \ldots, x_{n}$, we find $t f_{x_{j}}=f_{x_{j}}, i=1, \ldots, n$. Therefore, we have $f_{x_{1}}=\cdots=f_{x_{n}}=0$, which contradicts to the assumption that $f$ has non-vanishing first partial derivatives. Consequently, we must have $h \neq 0$. 
By taking the partial derivatives of (2.1) with respect to $x_{1}, \ldots, x_{n}$, respectively, we obtain

$$
\begin{gathered}
x_{1} f_{x_{1} x_{1}}+x_{2} f_{x_{1} x_{2}}+\cdots+x_{n} f_{x_{1} x_{n}}=(h-1) f_{x_{1}}, \\
x_{1} f_{x_{1} x_{2}}+x_{2} f_{x_{2} x_{2}}+\cdots+x_{n} f_{x_{2} x_{n}}=(h-1) f_{x_{2}}, \\
\vdots \\
x_{1} f_{x_{1} x_{n}}+x_{2} f_{x_{2} x_{n}}+\cdots+x_{n} f_{x_{n} x_{n}}=(h-1) f_{x_{n}} .
\end{gathered}
$$

Assume that the production function $f$ satisfies the constant elasticity of substitution property. Then we obtain (1.5) for some nonzero constant $\sigma$. Therefore, we have the following equations from (1.4):

$$
2 f_{x_{i}} f_{x_{j}} f_{x_{i} x_{j}}-f_{x_{j}}^{2} f_{x_{i} x_{i}}-f_{x_{i}}^{2} f_{x_{j} x_{j}}=\frac{\left(x_{i} f_{x_{i}}+x_{j} f_{x_{j}}\right) f_{x_{i}} f_{x_{j}}}{\sigma x_{i} x_{j}}
$$

for $1 \leq i<j \leq n$.

It follows from (2.3) that

$$
f_{x_{i} x_{j}}=\frac{\left(x_{i} f_{x_{i}}+x_{j} f_{x_{j}}\right) f_{x_{i}} f_{x_{j}}+\sigma x_{i} x_{j}\left(f_{x_{i} x_{i}} f_{x_{j}}^{2}+f_{x_{j} x_{j}} f_{x_{i}}^{2}\right)}{2 \sigma x_{i} x_{j} f_{x_{i}} f_{x_{j}}}
$$

for $1 \leq i<j \leq n$.

After substituting (2.4) into (2.2) and after solving the resulting equations for $f_{x_{i} x_{i}}, i=$ $1, \ldots, n$, we obtain

$$
f_{x_{i} x_{i}}=\left(\frac{1+(h-1) \sigma}{h \sigma} \cdot \frac{f_{x_{i}}}{f}-\frac{1}{\sigma x_{i}}\right) f_{x_{i}}, i=1, \ldots, n,
$$

by virtue of (2.1). Thus, after substituting (2.5) into (2.4) we get

$$
f_{x_{i} x_{j}}=\left(\frac{1+(h-1) \sigma}{h \sigma}\right) \frac{f_{x_{i}} f_{x_{j}}}{f}, 1 \leq i<j \leq n .
$$

Now, we divide the proof into two separate cases.

Case (a): $\sigma=1$. In this case, system (2.5) and (2.6) become

$$
\begin{aligned}
& f_{x_{i} x_{i}}=f_{x_{i}}\left(\frac{f_{x_{i}}}{f}-\frac{1}{x_{i}}\right), i=1, \ldots, n, \\
& f_{x_{i} x_{j}}=\frac{f_{x_{i}} f_{x_{j}}}{f}, 1 \leq i \neq j \leq n .
\end{aligned}
$$

After solving the second order differential equation given by (2.7) with $i=1$, we find

$$
f=\lambda\left(x_{2}, \ldots, x_{n}\right)\left(x_{1}\right)^{\mu\left(x_{2}, \ldots, x_{n}\right)}
$$


for some functions $\lambda\left(x_{2}, \ldots, x_{n}\right)$ and $\mu\left(x_{2}, \ldots, x_{n}\right)$.

By substituting (2.9) into the second order differential equation given by (2.8) with $i=1$ and $j \in\{2, \ldots, n\}$, we get

$$
\mu_{x_{2}}=\cdots=\mu_{x_{n}}=0 .
$$

Thus, in views of $f_{x_{1}} \neq 0$, the function $\mu$ is a nonzero constant, said $\alpha_{1}$. Hence (2.9) becomes

$$
f=\lambda\left(x_{2}, \ldots, x_{n}\right) x_{1}^{\alpha_{1}} .
$$

After substituting (2.10) into (2.7) with $i=2$, we obtain the following second order differential equation for $\lambda$ :

$$
\lambda_{x_{2} x_{2}}=\lambda_{x_{2}}\left(\frac{\lambda_{x_{2}}}{\lambda}-\frac{1}{x_{2}}\right)
$$

After solving this differential equation, we get

$$
\lambda=\varphi\left(x_{3}, \ldots, x_{n}\right)\left(x_{2}\right)^{\eta\left(x_{3}, \ldots, x_{n}\right)}
$$

for some functions $\varphi\left(x_{3}, \ldots, x_{n}\right)$ and $\eta\left(x_{3}, \ldots, x_{n}\right)$. By combining this with (2.10) we obtain

$$
f=\varphi\left(x_{3}, \ldots, x_{n}\right)\left(x_{2}\right)^{\eta\left(x_{3}, \ldots, x_{n}\right)} x_{1}^{\alpha_{1}} .
$$

Now, after substituting (2.12) into (2.8) with $i=2$ and $j \in\{3, \ldots, n\}$ and after applying $f_{x_{2}} \neq 0$, we conclude that $\eta\left(x_{3}, \ldots, x_{n}\right)$ is a nonzero constant, say $\alpha_{2}$. Therefore (2.12) becomes

$$
f=\varphi\left(x_{3}, \ldots, x_{n}\right) x_{1}^{\alpha_{1}} x_{2}^{\alpha_{2}}
$$

By continuing such procedure $n-1$ times, we derive that

$$
f=b x_{1}^{\alpha_{1}} \cdots x_{n}^{\alpha_{n}}
$$

for some positive real number $b$ and nonzero real numbers $\alpha_{i}, i=1, \ldots, n$, with $\sum_{i=1}^{n} \alpha_{i}=h$. Consequently, the production function $f$ is a generalized Cobb-Douglas production function.

Case (b): $\sigma \neq 1$. After solving the second order differential equation given by (2.5) with $i=1$, we get

$$
f=u\left(x_{2}, \ldots, x_{n}\right)\left(x_{1}^{\frac{\sigma-1}{\sigma}}+v\left(x_{2}, \ldots, x_{n}\right)\right)^{\frac{h \sigma}{\sigma-1}}
$$

for some functions $u\left(x_{2}, \ldots, x_{n}\right)$ and $v\left(x_{2}, \ldots, x_{n}\right)$.

By substituting (2.15) into the second differential equations given by (2.6) with $i=1$ and $j \in\{2, \ldots, n\}$, we derive that

$$
u_{x_{2}}=\cdots=u_{x_{n}}=0
$$


Thus $u$ is a constant. Therefore we may put $u=b a_{1}^{\frac{\sigma-1}{\sigma}}$ for some positive real numbers $b$ and $a_{1}$. Consequently, (2.15) becomes

$$
f=b\left(a_{1}^{\frac{\sigma-1}{\sigma}} x_{1}^{\frac{\sigma-1}{\sigma}}+v\left(x_{2}, \ldots, x_{n}\right)\right)^{\frac{h \sigma}{\sigma-1}} .
$$

Now, after substituting (2.16) into the differential equations given by (2.6) with $2 \leq i<j \leq n$, we find $v_{x_{i} x_{j}}=0$, which imply that

$$
v=v^{(2)}\left(x_{2}\right)+\cdots+v^{(n)}\left(x_{n}\right)
$$

for some non-constant functions $v^{(2)}\left(x_{2}\right), \ldots, v^{(n)}\left(x_{n}\right)$. Therefore, by combining this with (2.16) we obtain

$$
f=b\left(a_{1}^{\frac{\sigma-1}{\sigma}} x_{1}^{\frac{\sigma-1}{\sigma}}+v^{(2)}\left(x_{2}\right)+\cdots+v^{(n)}\left(x_{n}\right)\right)^{\frac{h \sigma}{\sigma-1}} .
$$

Now, by substituting this into the second order differential equations given by (2.5) with $i=$ $2, \ldots, n$, we conclude that $v^{(i)}\left(x_{i}\right) i=2, \ldots, n$, are of the form:

$$
v^{(i)}\left(x_{i}\right)=a_{i}^{\frac{\sigma-1}{\sigma}} x_{i}^{\frac{\sigma-1}{\sigma}}
$$

for some positive real numbers $a_{2}, \ldots, a_{n}$. Consequently, the production function is the ACMS function defined by (1.7) with $\rho=\frac{\sigma-1}{\sigma}$.

\section{Closing Remarks}

A production function is called quasi-sum if there are continuous strict monotone functions $g_{i}: \mathbb{R}_{+} \rightarrow \mathbb{R}, i=1, \ldots, n$, and there exist an interval $I \subset \mathbb{R}$ of positive length and a continuous strict monotone function $g: I \rightarrow \mathbb{R}_{+}$such that for each $\mathbf{x} \in \mathbb{R}_{+}^{n}$ we have $g_{1}\left(x_{1}\right)+\cdots+g_{n}\left(x_{n}\right) \in$ $I$ and

$$
f(\mathbf{x})=g\left(g_{1}\left(x_{1}\right)+\cdots+g_{n}\left(x_{n}\right)\right) .
$$

The justification for studying production functions of quasi-sum form is that these functions appear as solutions of the general bisymmetry equation and they are related to the problem of consistent aggregation (cf. [1]).

The generalized Cobb-Douglas production functions and the generalized ACMS production functions have been characterized by the homogeneity of some degree and quasi-sum form by W. Eichorn [9], B. Nyul [15] and F. Stehling [16].

Recently, several geometric results on $h$-homogeneous and quasi-sum production functions have been obtained by B.-Y. Chen, A. D. Vîlcu and G. E. Vîlcu using production hypersurfaces $[4,5,6,17,18]$. Several applications of these geometric results to generalized CobbDouglas' and ACMS production functions can be found in these papers.

Concerning the history on production functions, we refer to S. K. Mishra [14]. 


\section{References}

[1] J. Aczél and G Maksa, Solution of the rectangular $m \times n$ generalized bisymmetry equation and of the problem of consistent aggregation, J. Math. Anal. Appl., 203(2) (1996), 104-126.

[2] R. G. Allen and J. R. Hicks, A Reconsideration of the Theory of Value, Pt. II, Economica, 1 (1934), $196-219$.

[3] K. J. Arrow, H. B. Chenery, B. S. Minhas and R. M. Solow, Capital-labor substitution and economic efficiency, Rev. Econom. Stat., 43 (1961), 225-250.

[4] B.-Y. Chen, On some geometric properties of h-homogeneous production function in microeconomics, Kragujevac J. Math., 35(3) (2011), 343-357.

[5] B.-Y. Chen, On some geometric properties of quasi-sum production models, J. Math. Anal. Appl., 392 (2012), 192-199.

[6] B.-Y. Chen and G. E. Vîlcu, Geometric classifications of homogeneous production functions, Math. Social Sci., (submitted).

[7] C. W. Cobb and P. H. Douglas, A theory of production, Amer. Econom. Rev., 18 (1928), 139-165.

[8] P. H. Douglas, The Cobb-Douglas production function once again: Its history, its testing, and some new empirical values, J. Polit. Econom., 84 (5) (1976), 903-916.

[9] W. Eichorn, Characterization of CES production functions by quasilinearity, in Production Theory (W. Eichorn, R. Henn, O. Opitz and R. W. Shephard eds.), Springer-Verlag, pp. 21-33, 1974.

[10] J. Filipe and G. Adams, The Estimation of the Cobb Douglas function, Eastern Econom. J., 31 (3) (2005), 427445.

[11] J. R. Hicks, Theory of Wages, London, Macmillan, 1932.

[12] L. Losonczi, Production functions having the CES property, Acta Math. Acad. Paedagog. Nyhái. (N.S.) 26(1) (2010), 113-125.

[13] D. McFadden, Constant Elasticity of Substitution Production Functions, The Review of Economic Studies, 30(2) (1963), 73-83.

[14] S. K. Mishra, A brief history of production functions, IUP J. Manage. Econom., 8(4) (2010), 6-34.

[15] B. Nyul, Production functions and their characterizations, Alk. Mat. Lapok, 26(2) (2009), 351-363.

[16] F. Stehling, Eine neue Charakterisierung der CD- und ACMS-Produktiosfunktionen, Operations ResearchVerfahren, 21 (1975), 222-238.

[17] A. D. Vîlcu and G. E. Vîlcu, On some geometric properties of the generalized CES production functions, Appl. Math. Comput., 218(1) (2011), 124-129,

[18] G. E. Vîlcu, A geometric perspective on the generalized Cobb-Douglas production functions, Appl. Math. Lett., 24(5) (2011), 777-783.

Department of Mathematics, Michigan State University, 619 Red Cedar Road, East Lansing, Michigan 48824-1027, USA.

E-mail: bychen@math.msu.edu 